\title{
HYBRID MATCHING OF UNCALIBRATED OMNIDIRECTIONAL AND PERSPECTIVE IMAGES
}

\author{
Luis Puig, J.J. Guerrero \\ DIIS-I3A, University of Zaragoza, Zaragoza, Spain \\ lpuig@unizar.es,jguerrer@unizar.es \\ Peter Sturm \\ INRIA Rhône-Alpes, Montbonnot, France \\ Peter.Sturm@inrialpes.fr
}

Keywords: Computer vision, matching omnidirectional images, hybrid epipolar geometry.

\begin{abstract}
This work presents an automatic hybrid matching of central catadioptric and perspective images. It is based on the hybrid epipolar geometry. The goal is to obtain correspondences between an omnidirectional image and a conventional perspective image taken from different points of view. Mixing both kind of images has multiple applications, since an omnidirectional image captures many information and perspective images are the simplest way of acquisition. Scale invariant features with a simple unwrapping are considered to help the initial putative matching. Then a robust technique gives an estimation of the hybrid fundamental matrix, to avoid outliers. Experimental results with real image pairs show the feasibility of that hybrid and difficult matching problem.
\end{abstract}

\section{INTRODUCTION}

Recently, a number of catadioptric camera designs have appeared. The catadioptric cameras combine lenses and mirrors to capture a wide, often panoramic field of view. It is advantageous to capture a wide field of view for the following reasons. First, a wide field of view eases the search for correspondences as the corresponding points do not disappear from the images so often. Second, a wide field of view helps to stabilize egomotion estimation algorithms, so that the rotation of the camera can be easily distinguished from its translation. Last but not least, almost complete reconstructions of a surrounding scene can be obtained from two panoramic images (Svoboda and Pajdla, 2002).

A hybrid image matching is a system capable of establish a relation between two or more images coming from different camera types. The combination of omnidirectional and perspective images is important in the sense of that a single omnidirectional image contains more complete description of the object or place it represents than a perspective image thanks to its wide field of view and its ability to unfold objects.

In general if we want to establish a relation between two or more views obtained from different cameras, they must have a common representation where they can be compared. We face this problem when we want to match omnidirectional images with perspective ones. Few authors have dealt with this problem and they followed different strategies. In (Menem and Pajdla, 2004) Plücker coordinates are used to map $\wp^{2}$ into $\wp^{5}$ where lines and conics are represented in 6-vectors and a geometrical relation can be established. A change of feature space is introduced in (Chen and Yang, 2005). The perspective image is partitioned into patches and then each patch is registered in the Haar feature space. These approaches have a major drawback, they require information about the geometry of the particular mirror used to get the images, which in most cases is not available. Besides they perform hard transformations over the image.

Another approach to deal with this problem is to establish a geometric relation between the images. Sturm (Sturm, 2002) develops this type of relation between multiple views of a static scene, where these views are obtained from para-catadioptric and perspective cameras.

We propose to explore a way to overcome these drawbacks avoiding the use of the so complex geometry of the mirror and the formulation of a geomet- 
ric model. We present an automatic hybrid matching approach with uncalibrated images using the hybrid epipolar geometry to establish a relation between omnidirectional and perspective images.

\section{HYBRID IMAGE MATCHING USING EPIPOLAR GEOMETRY}

To deal with the problem of the robust hybrid matching a common strategy is to establish a geometrical relation between the views of the 3D scene. We have selected a strategy that does not require any information about the mirror. A geometrical approach which encapsulates the projective geometry between two views is used. Epipolar geometry (EG) is the intrinsic projective geometry between two views. It is independent of the scene structure, and only depends on the cameras' internal parameters and relative pose (Hartley and Zisserman, 2000). This approach needs pairs of putative corresponding points between the views. In this work we use the SIFT descriptor (Lowe, 2004).

Sturm proposes a hybrid epipolar geometry, where a point in the perspective image is mapped to its corresponding epipolar conic in the omnidirectional image. Recently Barreto and Daniliidis (Barreto and Daniilidis, 2006) have exposed a more general scheme where they compare the mixture of central cameras, including pin-hole, hyperbolic and parabolic mirrors in catadioptric systems and perspective cameras with radial distortion.

The fundamental matrix $\mathbf{F}$ encapsulates the epipolar geometry. The dimension of this matrix depends on the image types we want to match. In the hybrid case we have two options, a $4 \times 3$ matrix in the case of para-catadioptric and perspective cameras or $6 \times 3$ in the case of hyperbolic mirror and perspective cameras in a catadioptric system. In (Barreto and Daniilidis, 2006) for this last case a $6 \times 6$ matrix is considered, which can result in a very difficult corresponding estimation problem.

\subsection{EG with Perspective and Catadioptric Cameras}

In general the relation between omnidirectional and perspective images with the fundamental matrix can be established by

$$
\hat{\mathbf{q}}_{c}^{T} \mathbf{F}_{c p} \mathbf{q}_{p}=0
$$

subscripts $p$ and $c$ denote perspective and catadioptric respectively.
From Eq.1 with known corresponding points between the two images, we can derive the hybrid fundamental matrix. Points in the perspective image are defined in common homogeneous coordinates. Points in the omnidirectional image are defined depending on the shape of the epipolar conic. The general representation for any shape of epipolar conic is a 6-vector. A special case where the shape of the conic is a circle the coordinate vector has four elements. These representations are called the "lifted coordinates" of a point in the omnidirectional image.

In the hybrid epipolar geometry points in the perspective image are mapped to its corresponding epipolar conic in the omnidirectional image. Conics can be represented in homogeneous coordinates as the product $\hat{\mathbf{q}}^{T} \mathbf{c}=0$, where $\hat{\mathbf{q}}^{T}$ represents the lifted coordinates of the omnifirectional point $\mathbf{q}$. In this work we have two representations for this point, one of them is the general homogeneus form of a conic, a 6 -vector $\hat{\mathbf{q}}=\left(q_{1}^{2}, q_{2}^{2}, q_{3}^{2}, q_{1} q_{2}, q_{1} q_{3}, q_{2} q_{3}\right)^{T}$. The other one constraints the shape of the conic to be a circle $\hat{\mathbf{q}}=\left(q_{1}^{2}+q_{2}^{2}, q_{1} q_{3}, q_{2} q_{3}, q_{3}^{2}\right)^{T}$. These representations are called the "lifted coordinates" of the omnidirectional point $\mathbf{q}$.

If the point in the omnidirectional image is represented with a 6 -vector lifted coordinates $\hat{\mathbf{q}}$, the fundamental matrix is $6 \times 3$ (F63) in such a way that $\mathbf{c} \sim \mathbf{F}_{c p} \mathbf{q}_{p}$. When the 4-vector lifted coordinates is used the fundamental matrix is $4 \times 3$ and the conic (circle) is obtained by the same product $\mathbf{c} \sim \mathbf{F}_{c p} \mathbf{q}_{p}$.

\subsection{Computation of the Hybrid Fundamental Matrix}

The algorithm used to compute the fundamental matrix is similar to the 8-point algorithm (Hartley and Zisserman, 2000) for the purely perspective case, with the difference that the points in the omnidirectional images are given in lifted coordinates.

The automatic computation of the fundamental matrix is summarized as follows:

1. Initial Matching. Scale invariant features are extracted in each image and matched based on their intensity neighborhood.

2. RANSAC robust estimation. Repeat for $n$ samples, where $n$ is determined adaptively:

(a) Select a random sample of $k$ corresponding points, where $k$ depends on what model we are using (if F43, $k=11$ or if F63, $k=17)^{1}$. Compute the hybrid fundamental matrix $\mathbf{F}_{c p}$ as described above.

\footnotetext{
${ }^{1}$ Matrices are up to scale, so we need the number of elements of the matrix minus one corresponding points.
} 
(b) Compute the distance $d$ for each putative correspondence, $d$ is the geometric distance from a point to its corresponding epipolar conic (Sturm and Gargallo, 2007).

(c) Compute the number of inliers consistent with $\mathbf{F}_{c p}$ by the number of correspondences for which $d<t$ pixels.

Choose the $\mathbf{F}_{c p}$ with the largest number of inliers.

The $\mathbf{F}_{c p}$ is used to eliminate outliers which are those point correspondences for which $d>t$.

\section{HYBRID EG EXPERIMENTS}

In this section we present experiments performed with real images obtained from two different omnidirectional cameras. The first one is a model composed by an unknown shape mirror and a perspective camera. The second one is composed by a hyperbolic mirror and a perspective camera.

The purpose of this experiment is to show the performance of this approach to compute the hybrid epipolar geometry in real images. We use 40 manually selected corresponding points to compute it. In order to measure the performance of $\mathbf{F}$ we calculate the geometric error from each point to its corresponding epipolar conic. Fig.1(a) shows the epipolar conics computed with the F43 matrix using the unknown shape mirror. Fig. 1(b) shows the epipolar conics computed with the F63 matrix using the hyperbolic mirror. The mean of distances from the points to their corresponding epipolar conics are 2.07 pixels and 0.95 pixels respectively.
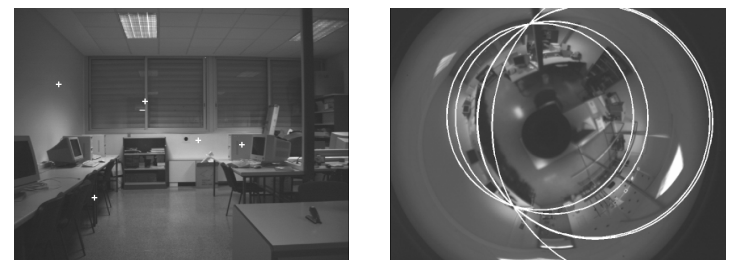

(a) Experiment with the unknown shape mirror using F43.
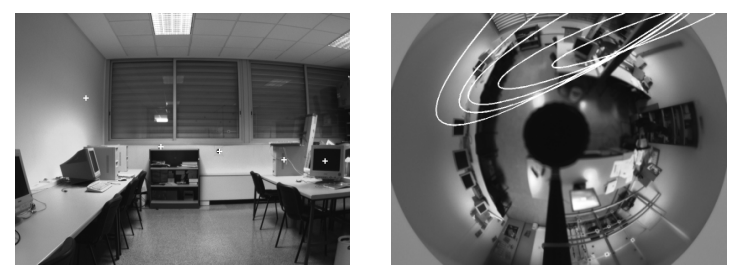

(b) Experiment with the hyperbolic using F63.

Figure 1: Experiments with real images using both mirrors and both hybrid fundamental matrices.

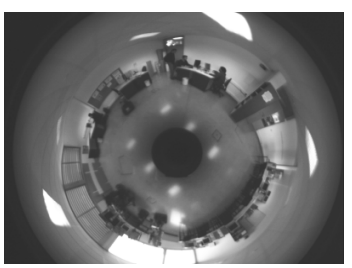

(a)

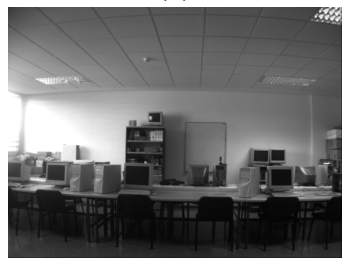

(c)

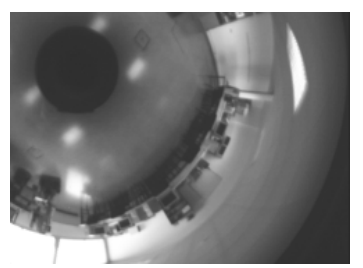

(b)

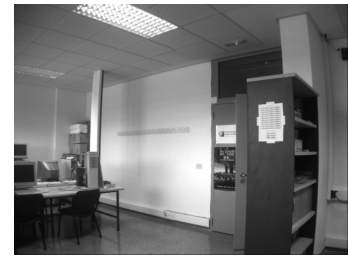

(d)
Figure 2: Set of images used to test the automatic approach. (a) Omnidirectional image. (b) An image cut from (a). (c) Perspective1 image. (d) Perspective2 image.

\section{AUTOMATIC MATCHING}

In this section we present some experiments performing automatically the matching between an omnidirectional and a perspective image. There exist approaches matching automatically omnidirectional images using scale invariant features (Murillo et al., 2007) which demonstrate a good performance, but it does not work with hybrid matching. An easy way to transform an omnidirectional image into a common environment with the perspective one is to unwrap the omnidirectional image, which consists in a mapping from Cartesian to polar coordinates.

The initial matching between the perspective and the unwrapped omnidirectional image has a considerable amount of inliers but also a majority of outliers. This scenario is ideal to use a technique like RANSAC where we have a set of possible correspondences of points useful to compute the hybrid epipolar geometry.

One omnidirectional image, a part of it and two perspective images, all of them uncalibrated, are used to perform the following experiment. We use the algorithm explained in section 2.2. Table 1 summarizes the results of this experiments giving the quantity of inliers and outliers in the initial and the robust matching. For example, in Experiment 1 we use images Fig.2(a) and Fig.2(c). The initial matching gives a $35 \%$ of inliers. After applying the robust estimation we obtain a $80 \%$ of inliers. Notice that just 2 inliers have been eliminated. Fig. 3 shows the final matches.

The results show that the epipolar geometry eliminates most of the outliers. For practical reasons in the estimation problem, we use the F43, the simplest model of the hybrid epipolar fundamental matrix. 


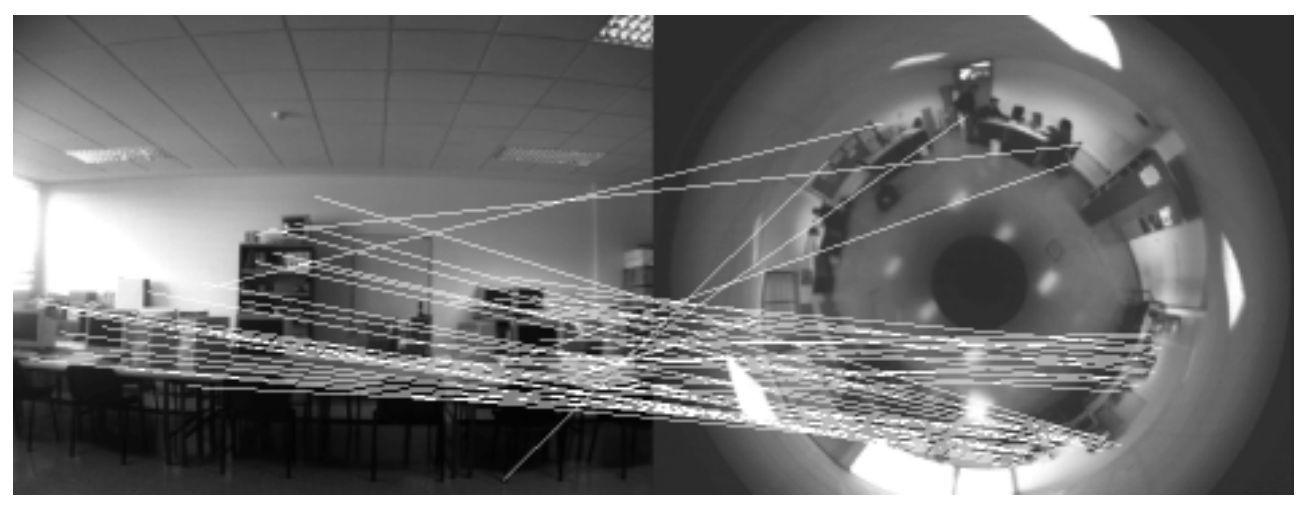

Figure 3: Matching between omnidirectional and perspective image using the unwrapping and the hybrid EG as a tool.

Table 1: Numerical results of the matches using the set of images.

\begin{tabular}{|l|c|c|c|c|}
\hline & Omni SIFT & Persp SIFT & $\begin{array}{c}\text { Initial Matches } \\
\text { (inliers/outliers) }\end{array}$ & $\begin{array}{c}\text { Robust EG matches } \\
\text { (inliers/outliers) }\end{array}$ \\
\hline Experiment 1 & 636 & 941 & $37 / 70$ & $35 / 9$ \\
\hline Experiment 2 & 636 & 1229 & $19 / 36$ & $16 / 11$ \\
\hline Experiment 3 & 246 & 941 & $36 / 11$ & $34 / 4$ \\
\hline Experiment 4 & 246 & 1229 & $20 / 14$ & $16 / 6$ \\
\hline
\end{tabular}

\section{CONCLUSIONS}

In this work we have presented an automatic widebaseline hybrid matching system using uncalibrated cameras. We have performed experiments using real images with different mirrors and two different ways to compute the hybrid fundamental matrix, a 6-vector generic model and a reduced 4 -vector model. The last one has demonstrated be useful in order to require fewer iterations to compute a robust fundamental matrix and having equal or even better performance than the 6-vector approach. We also prove that an easy polar transformation can be a useful tool to perform a basic matching between omnidirectional and perspective images. Finally the robust automatic matching proved its efficiency to match an omnidirectional image and a perspective image, both uncalibrated.

\section{ACKNOWLEDGEMENTS}

This work was supported by project NERO DPI2006 07928 and DGA(CONSI+D)/CAI.

\section{REFERENCES}

Barreto, J. P. and Daniilidis, K. (2006). Epipolar geometry of central projection systems using veronese maps.
In CVPR '06: Proceedings of the 2006 IEEE Computer Society Conference on Computer Vision and Pattern Recognition, pages 1258-1265, Washington, DC, USA. IEEE Computer Society.

Chen, D. and Yang, J. (2005). Image registration with uncalibrated cameras in hybrid vision systems. In WACV/MOTION, pages 427-432.

Hartley, R. I. and Zisserman, A. (2000). Multiple View Geometry in Computer Vision. Cambridge University Press, ISBN: 0521623049.

Lowe, D. (2004). Distinctive image features from scaleinvariant keypoints. In International Journal of Computer Vision, volume 20, pages 91-110.

Menem, M. and Pajdla, T. (2004). Constraints on perspective images and circular panoramas. In Andreas, H., Barman, S., and Ellis, T., editors, BMVC 2004: Proceedings of the 15th British Machine Vision Conference, London, UK. BMVA, British Machine Vision Association.

Murillo, A. C., Guerrero, J. J., and Sagües, C. (2007). Surf features for efficient robot localization with omnidirectional images. In 2007 IEEE International Conference on Robotics and Automatio, Roma.

Sturm, P. (2002). Mixing catadioptric and perspective cameras. In Workshop on Omnidirectional Vision, Copenhagen, Denmark, pages 37-44.

Sturm, P. and Gargallo, P. (2007). Conic fitting using the geometric distance. In Proceedings of the Asian Conference on Computer Vision, Tokyo, Japan. Springer.

Svoboda, T. and Pajdla, T. (2002). Epipolar geometry for central catadioptric cameras. Int. J. Comput. Vision, 49(1):23-37. 